\title{
Individual differences in working memory capacity and divided attention in dichotic listening
}

\author{
Gregory J. H. Colflesh \\ University of Illinois, Chicago, Illinois \\ AND \\ ANDREw R. A. Conway \\ Princeton University, Princeton, New Jersey
}

\begin{abstract}
The controlled attention theory of working memory suggests that individuals with greater working memory capacity (WMC) are better able to control or focus their attention than individuals with lesser WMC. This relationship has been observed in a number of selective attention paradigms including a dichotic listening task (Conway, Cowan, \& Bunting, 2001) in which participants were required to shadow words presented to one ear and ignore words presented to the other ear. Conway et al. found that when the participant's name was presented to the ignored ear, $65 \%$ of participants with low WMC reported hearing their name, compared to only $20 \%$ of participants with high WMC, suggesting greater selective attention on the part of high WMC participants. In the present study, individual differences in divided attention were examined in a dichotic listening task, in which participants shadowed one message and listened for their own name in the other message. Here we find that $66.7 \%$ of high WMC and $34.5 \%$ of low WMC participants detected their name. These results suggest that as WMC capacity increases, so does the ability to control the focus of attention, with high WMC participants being able to flexibly "zoom in" or "zoom out" depending on task demands.
\end{abstract}

Since Baddeley and Hitch (1974) first published their seminal chapter on working memory (WM), many theories regarding the construct have been proposed. One of the more widely supported theories, particularly when applied to individual differences in WM, is the controlled attention theory of WM (Engle \& Kane, 2004; Kane, Bleckley, Conway, \& Engle, 2001; Kane \& Engle, 2002), according to which there is a domain-general component of WM responsible for guiding attention as well as domain-specific components responsible for maintenance of task-relevant information. Individuals who score high on tests of working memory capacity (WMC) therefore may do so because of greater controlled attention and/or because of better use of domain-specific skills and strategies to aid maintenance. Engle and colleagues have argued that the domain-general controlled attention ability is related to both higher-level cognition, such as fluid intelligence, reading comprehension, and problem solving, and lower level cognition, such as performance of simple visual and auditory attention tasks that require cognitive control (for a review, see Engle \& Kane, 2004).

To date, all of the experiments that have demonstrated a relationship between WMC and lower level cognition have come from selective attention paradigms. For example, Kane et al. (2001) demonstrated the relationship using a visual orienting paradigm (i.e., pro- and antisaccade tasks). They found no difference between high and low WMC participants in the prosaccade task but high WMC participants performed significantly better than low WMC participants on the antisaccade task (see also Unsworth, Schrock, \& Engle, 2004). The relationship between WMC and controlled attention was also observed in the Stroop task (Kane \& Engle, 2003). In conditions that demanded less attention to maintain the task goal (i.e., when the ink color and the color word matched on either $0 \%$ or $100 \%$ of the trials) there was no difference in performance between high and low WMC participants; however, when the task demanded more attention to maintain the task goal (i.e., when the ink color and the color word matched on $75 \%$ of the trials) high WMC participants performed significantly better than low WMC participants.

Most relevant to the present project, the relationship between WMC and controlled attention has also been observed in the dichotic listening paradigm (Conway, Cowan, \& Bunting, 2001). In a dichotic listening task, two messages are presented simultaneously, one to each ear. Conway et al. instructed participants to shadow the words presented to one ear while ignoring the words presented to the other ear, hence participants were required to use selective attention. Each participant's own name was presented to the ignored message, and Conway et al. found that $65 \%$ of participants with low WMC reported 
hearing their name, while only $20 \%$ of participants with high WMC reported hearing their name. Participants with greater WMC were better able to control their attention and focus on the relevant message, thus hearing their name less often.

Even though the above cited evidence supports the controlled attention view of WM, one limitation of previous research is that all the experiments tested selective attention only. It is therefore an open question as to whether WMC will be related to performance in a divided attention paradigm. Cowan (2005) suggests that the spotlight (or focus) of attention is flexible, and that individuals differ in their ability to adapt the focus of attention according to task goals. If the task requires selective attention, then the spotlight of attention should be zoomed in and only the information relevant to the task should be processed. If the task requires attention to be divided, then the spotlight of attention should be zoomed out accordingly so that all information relevant to the task may be processed. However, the nature of individual differences in the flexibility of the spotlight of attention is unknown. It may be that higher WMC leads only to being able to focus (i.e., remain zoomed in). In contrast, it may be that higher WMC is related to the ability to flexibly adjust attention (i.e., zoom in or out depending on task demands). The nature of the selective attention tasks used in previous individual differences experiments does not allow us to examine flexibility because participants were expected to continuously focus their attention; therefore, divided attention tasks will allow us to explore flexibility because participants need to "zoom out" to perform the task appropriately.

The motivation for the present experiment was to explore whether WMC is related to performance in a divided attention paradigm. To explore this, participants performed a dichotic listening task similar to that used by Conway et al. (2001) except participants had to divided their attention between the two messages. The results of previous studies (Kane et al., 2001; Kane \& Engle, 2003) suggest that the level of attentional demand that a task has may influence the relationship that WMC plays in the completion of the task. Therefore, in the present experiment we manipulated attentional demand in two ways. First, we required subjects to perform two versions of the dichotic listening task, once while shadowing (i.e., high attentional demand) and once while not shadowing (i.e., low attentional demand). Second, we manipulated the signal-to-noise ratio (SNR) between the two messages that were presented, such that the more relevant message was either quieter than, the same volume as, or louder than the message with the name (i.e., more attentionally demanding to less attentionally demanding, respectively). If WMC is related to the ability to control attention, then a relationship should be observed between WMC and performance on the divided attention task, such that as WMC increases, performance on the attention task should increase. However, similar to the results obtained in the aforementioned selective attention experiments, this difference should only be observed in situations where the task is more attentionally demanding. Therefore, even though dividing attention while not shadowing does re- quire attention, it has a low attentional demand and is not likely to require the control of attention to complete the task and it is predicted that WMC will not impact the outcome, whereas dividing attention while shadowing has a high attentional demand and is expected to require a greater control of attention to complete the task and it is predicted that WMC will have a significant effect on the outcome.

\section{METHOD}

\section{Participants}

The sample comprised 118 (71 male, 47 female) undergraduate students from the University of Illinois at Chicago subject pool who received course credit for participating. The participants were native English speakers, with normal hearing, and monosyllabic first names. All participants were screened for WMC prior to performing the dichotic listening task.

\section{Working Memory Screening}

The procedures used to measure WMC were adapted from versions of the operation span task and reading span task used by Kane, Hambrick, Tuholski, Wilhelm, Payne, and Engle (2004). We followed the administration and scoring guidelines recommended by Conway et al. (2005). To be consistent with the Conway et al. terminology, each task consists of "items" that vary in difficulty, which is manipulated by varying the number of "elements" per item. This terminology is consistent with psychometric lingo where a test (or task) consists of items that vary in difficulty. Each task, operation span and reading span, consisted of 12 items. The number of elements per item varied from 2 to 5 , with three items at each level of difficulty.

In the operation span task, each element consisted of a mathematical operation and a word (e.g., IS [7*3] $+4=21$ ? SNOW). The participant's task was to read the math problem aloud, say "yes" or "no" to indicate whether the given answer was correct or incorrect and then say the word. After all the elements in an item were presented the participant was required to write the words in correct serial order. The difficulty of the items was randomized such that the number of elements was unpredictable at the outset of an item.

In the reading span task, each element consisted of a sentence, followed by a letter (e.g., Andy was stopped by the policeman because he crossed the yellow heaven. ? R). The participant's task was to say the sentence aloud, respond "yes" or "no" to indicate whether the sentence made sense and then say the letter. As with operation span, after all the elements in an item were presented the participant was required to write the letters in correct serial order and the difficulty of the items was randomized such that the number of elements was unpredictable at the outset of an item.

Proportion scores were computed for each item by dividing the number of correctly recalled stimuli by the total number of stimuli in that item (e.g., if the participant correctly recalled two of four words, then the score for that item would be .5). The total proportion score was simply the average of the item proportion scores multiplied by 100 to yield a scale of $0-100$. Finally, an overall proportion score for WMC was computed by averaging the total proportion scores for operation span and reading span.

\section{Divided Attention Procedure}

Each participant performed two tasks: divided attention-shadow and divided attention-no shadow. The order of the tasks was counterbalanced. Participants completed the tasks at one of three SNRs: $-8,0$, and +8 (i.e., the more relevant message was presented either $-8,0$, or +8 decibels compared to the less relevant message). Participants completed the two tasks at only 1 SNR and SNR was randomly assigned. In the divided attention-shadow condition, participants were instructed to listen to the more relevant message (presented to the right ear) and to repeat (shadow) each word as soon 
Table 1

Descriptive Statistics of WMC, Shadowing Errors, and Name Detection

\begin{tabular}{|c|c|c|c|}
\hline Measure & $M$ & $S D$ & $N$ \\
\hline WMC & 66.14 & 12.23 & 118 \\
\hline \multicolumn{4}{|l|}{ Shadowing Errors } \\
\hline $\mathrm{SNR}-8$ & 54.36 & 28.57 & 39 \\
\hline SNR 0 & 50.15 & 27.82 & 40 \\
\hline $\mathrm{SNR}+8$ & 35.97 & 26.66 & 39 \\
\hline Total & 46.86 & 28.56 & 118 \\
\hline \multicolumn{4}{|l|}{ Name Detection } \\
\hline Shadow & 50.00 & 50.21 & 118 \\
\hline No shadow & 85.60 & 35.27 & 118 \\
\hline
\end{tabular}

Note-WMC, working memory capacity; SNR, signal-to-noise ratio.

as it was heard, making as few errors as possible. Participants were told that their name would be presented in the unshadowed message and upon hearing their name they should press the space bar on the computer keyboard. Participants were asked to continue shadowing until all sound on the more relevant message had stopped. While participants were shadowing, the experimenter was seated at a separate table in the room and recorded shadowing errors. Prior to the divided attention-no shadow condition participants were told that their name would be presented somewhere within the two messages they would listen to, and upon hearing their name, they should press the space bar. The name was always presented to the left ear. Unlike selective attention dichotic listening experiments, participants were not asked at the end of the experiment whether they heard their name. This was not necessary as pressing the space bar allowed for assessment of the participants hearing their name.

\section{Divided Attention Stimuli}

The auditory stimuli were digitized onto a computer at a sampling rate of $22 \mathrm{kHz}$ and dynamic range of 16 bits. There were three sets of auditory stimuli, each of which comprised a more relevant and a less relevant message. They were presented through stereo headphones, with each set being presented at one of three SNRs. The stimulus lists for the divided attention-no shadow task were the same as in the shadowed task; however, participants were presented with a list that they had not shadowed, thus each separate list appeared unique to the participant. The more relevant message consisted of 180 unrelated monosyllabic words (i.e., did not form a coherent text) recorded in a monotone female voice at a rate of 60 words per min and lasted $3 \mathrm{~min}$. The less relevant message contained 150 unrelated monosyllabic words recorded in a monotone male voice at a rate of 60 words per min and lasted $2.5 \mathrm{~min}$. The onset of the more relevant message began 30 words prior to the presentation of the less relevant stimuli, allowing participants to become acquainted with the more relevant message without distraction. The onsets of the words in the two channels were synchronized. The order of the words in each SNR group $(-8,0,+8)$ was identical across participants except for the names. Participants' first names were randomly inserted into the less relevant message in place of a word, either early, at $1 \mathrm{~min} 30 \mathrm{sec}$, or late, at $2 \mathrm{~min} 30 \mathrm{sec}$.

\section{RESULTS}

\section{Descriptive Statistics}

Descriptive statistics for all measures are reported in Table 1. The distribution of scores for the span tasks are similar to those obtained in others labs (see Conway et al., 2005; Kane et al., 2004). As expected, the percentage of participants who reported hearing their name was greater in the no-shadowing condition $(M=85.6 \%)$ than in the shadowing condition $(M=50 \%)$ [McNemar test $(1, N=$ $118)=31.5, p<.05]$. Not surprisingly, these percentages are higher than the $33 \%$ that is typically observed in selective attention versions of this task (Conway et al., 2001; Moray, 1959; Wood \& Cowan, 1995).

\section{Name Detection}

Two separate (shadow, no shadow) hierarchical binary logistic regression analyses were conducted to assess the role that WMC and SNR play on the likelihood of a participant hearing their name. For all analyses, WMC and SNR were entered in the first step of the regression analysis and the WMC $\times$ SNR interaction was entered in the second step. WMC was centered. SNR was treated as a continuous variable, coded in order of decreasing attentional demand (i.e., $-8,0$, and +8 , coded as 0,1 , and 2 , respectively).

The results of the shadow analysis indicated that there was a significant main effect due to WMC (Wald $=3.85$, $p<.05$; OR $=1.03$ ). Therefore, for each 1 unit increase in WMC, participants were 3\% more likely to hear their name. More concretely, a one standard deviation increase in WMC (12 points) means that a participant was $36 \%$ more likely to detect their name. Both SNR and the interaction were not significant (Walds $=.15$ and .24 , respectively).

The same results are presented by WMC category (see Figure 1), to better compare the present results with those in the parallel selective attention version of this task reported by Conway et al. (2001). Conway et al. compared only two categories of subjects: high span and low span. High spans had WMC scores that fell in the upper quartile of a large distribution of WMC scores $(M=24.85$; range of 17-44) and low spans had WMC scores that fell in the lower quartile $(M=8.22$; range of $6-12)$. In the present project we collected dichotic listening data from the entire range of WMC; therefore, to compare our results with those from Conway et al., we constructed two WMC categories: high WMC (upper quartile) $(M=82.16$; range of 75-91.04) and low WMC (lower quartile) $(M=50.81$; range of 31.53-57.99). ${ }^{1}$ With these boundaries, $66.7 \%$ of high WMC participants and $34.5 \%$ of low WMC participants heard their name. This pattern of results is particularly striking when compared to the results of Conway et al. (2001), who observed the opposite pattern in a selective attention task; $65 \%$ of the low WMC participants reported hearing their name and only $20 \%$ of the high WMC participants reported hearing their name.

The results of the no shadow analysis indicated that neither WMC, SNR, nor the interaction were significant (Walds $=.10,1.70$, and .30 , respectively). The results broken down by WMC category are presented in Figure 1. As predicted, WMC did not have an effect on hearing the name in the no shadow task.

\section{Shadowing Errors}

A hierarchical linear regression was conducted to examine the relationship between WMC, SNR, and shadowing errors. The results of the initial model accounted for a significant proportion of variance [adjusted $R^{2}=.08$; $F(2,115)=6.35, p<.05]$. SNR was a significant predictor of the number of shadowing errors $(\beta=-.24, t=$ $-2.64, p<.05)$, whereas WMC was only marginally sig- 


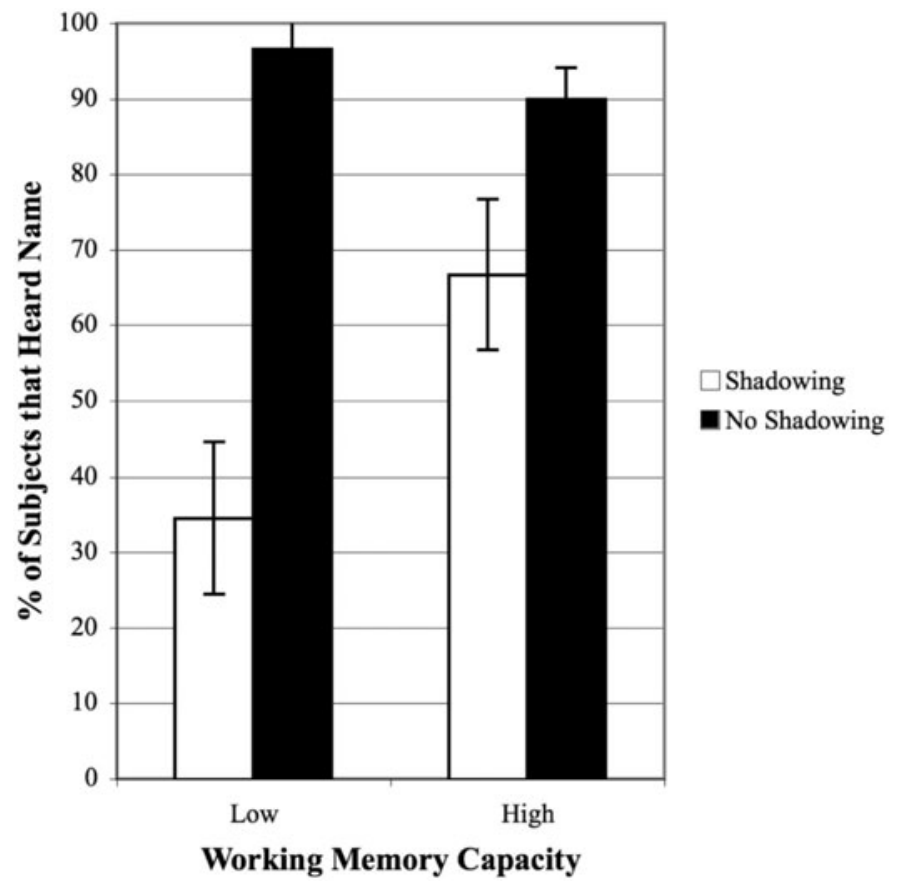

Figure 1. The percentage of high WMC and low WMC participants that heard their name. Error bars represent standard errors.

nificant $(\beta=-.18, t=-1.97, p=.051)$. There was not a significant change in the amount of variance predicted with the addition of interaction term $\left(R^{2}\right.$ change $=.00$, $F$ change $=.33, p>.05)$. These results suggest that participants with greater WMC made fewer errors than participants with lesser WMC. Therefore, it is unlikely that the larger proportion of participants with greater WMC hearing their name is due to higher WMC participants neglecting the shadowing task.

\section{DISCUSSION}

The results of this experiment provide additional yet unique support for the controlled attention theory of WM. Previously, the relationship between WMC and the ability to control attention was only supported by research on selective attention. Here we demonstrate that individuals with greater WMC are also better able to divide attention than are individuals with lesser WMC. Not surprisingly, when the divided attention task was less demanding (i.e., no shadowing), there was not a significant effect of WMC on task performance; however, when the task was demanding (i.e., shadowing), there was a significant effect of WMC, such that as WMC increased, so did performance on the attention task, as indexed by likelihood of name detection and shadowing errors. Unfortunately, the SNR manipulation did not impact the likelihood of name detection. As a result, it is not surprising that SNR did not interact with WMC to predict name detection.

The results of the no-shadowing divided attention task likely represent a ceiling effect, which makes it difficult to determine the role of WMC. However, the prediction of this effect was theoretically motivated. As discussed earlier, WMC is not related to performance in all situations that require attention, but seems to be selectively related to performance in attention tasks that place greater demands on executive attention. While the ceiling effect in the no shadowing condition does temper our conclusion about the attentional demand manipulations, it is reassuring to observe that low span participants were able to detect their names in this condition because it rules out the uninteresting interpretation that low span participants are simply unable to follow directions.

Comparing the results of the present study to the results obtained by Conway et al. (2001) presents a bit of a paradox, particularly when considering the performance of low span participants. ${ }^{2}$ Conway et al. found that in the selective attention dichotic listening task $65 \%$ of low spans and only $20 \%$ of high spans reported hearing their name. The results of the present experiment revealed an opposite pattern, such that in the divided attention, shadowing task, $66.7 \%$ of high spans and $34.5 \%$ of low spans heard their name. The interpretation of the high span data is straightforward, i.e., these participants are better able to adjust the focus of attention according to task goals. They focus attention in the selective attention task, thus hearing their name less often, and they split their attention in the divided attention task, thus hearing their name more often.

But what does one make of the performance of low span participants? They were actually less likely to hear their name when they were told to listen for it than when they had no idea that it was coming! How do we reconcile these results? A critical difference between Conway et al.'s selective attention task and the present divided attention task 
is the way in which one's name was detected. In the selective attention paradigm subjects were asked, after shadowing, if they thought they heard their name in the ignored message. In the divided attention task, participants were required to press the space bar immediately after detecting their name. Thus, in the selective attention task low spans are more susceptible to attentional capture by a salient distractor and in the divided attention task they are less able to coordinate the demands of shadowing, listening for their name, and signaling name detection.

These results are consistent with Cowan's (2005) adjustable-attention theory of individual differences in WMC and fluid intelligence. Cowan argues that greater WMC, as well as greater fluid intelligence, is associated with the ability to adjust attention according to task goals. Individuals with greater WMC and greater fluid intelligence are better able to configure attention to meet task goals. Previous research on selective attention supports the notion that individuals with greater WMC are better able to focus attention and avoid distraction and the present results support the notion that individuals with greater WMC are better able to "zoom out" and divide attention. There is also evidence to support the notion that individuals with greater WMC are better able to configure visual attention, discontiguously rather than contiguously, depending on which configuration optimizes task performance (Bleckley, Durso, Crutchfield, Engle, \& Khanna, 2003). However, what remains a question for future research is whether greater WMC is also related to the ability to dynamically adapt attention to meet task demands, rather than simply the ability to set the focus of attention according to given task goals. In other words, might high spans be able to dynamically adapt their focus of attention as task demands change or might they be more resistant to change their focus once it is set? This question awaits future research.

\section{AUTHOR NOTE}

This research was submitted to the University of Illinois at Chicago in partial fulfillment of the requirements of the Master of Arts degree by G.J.H.C. We thank Jennifer Wiley and James W. Pellegrino for their helpful comments during the preparation of the manuscript. Correspondence concerning this article should be addressed to G. J. H. Colflesh, University of Illinois Chicago, Department of Psychology (M/C 285), 1007 West Harrison Street, Chicago, IL 60607 (e-mail: colflesh@uic.edu).

\section{REFERENCES}

BAdDeley, A., \& Hitch, G. J. (1974). Working memory. In G. H. Bower (Ed.), The psychology of learning and motivation (Vol. 8, pp. 47-90). New York: Academic Press.

Bleckley, M. K., Durso, F. T., Crutchfield, J. M., Engle, R. W., \& Khanna, M. M. (2003). Individual differences in working memory capacity predict visual attention allocation. Psychonomic Bulletin \& Review, 10, 884-889.
Conway, A. R. A., Cowan, N., \& Bunting, M. F. (2001). The cocktail party phenomenon revisited: The importance of working memory capacity. Psychonomic Bulletin \& Review, 8, 331-335.

Conway, A. R. A., Kane, M. J., Bunting, M. F., Hambrick, D. Z., Wilhelm, O., \& Engle, R. W. (2005). Working memory span tasks: A methodological review and user's guide. Psychonomic Bulletin \& Review, 12, 769-786.

CoWAN, N. (2005). Understanding intelligence: A summary and an adjustable-attention hypothesis. In O. Wilhelm \& R. W. Engle (Eds.), Handbook of understanding and measuring intelligence (pp. 469488). London: Sage.

Engle, R. W., \& Kane, M. J. (2004). Executive attention, working memory capacity, and a two-factor theory of cognitive control. In B. Ross (Ed.), The psychology of learning and motivation (Vol. 44, pp. 145-199). New York: Elsevier.

Kane, M. J., Bleckley, M. K., Conway, A. R. A., \& Engle, R. W. (2001). A controlled-attention view of working memory capacity: Individual differences in memory span and the control of visual orienting. Journal of Experimental Psychology: General, 130, 169-183.

Kane, M. J., \& Engle, R. W. (2002). The role of prefrontal cortex in working-memory capacity, executive attention, and general fluid intelligence: An individual differences perspective. Psychonomic Bulletin \& Review, 9, 637-671.

KANE, M. J., \& ENGLE, R. W. (2003). Working memory capacity and the control of attention: The contributions of goal neglect, response competition, and task set to Stroop interference. Journal of Experimental Psychology: General, 132, 47-70.

Kane, M. J., Hambrick, D. Z., Tuholski, S. W., Wilhelm, O., Payne, T. W., \& ENGLE, R. W. (2004). The generality of working memory capacity: A latent variable approach to verbal and visuospatial memory span and reasoning. Journal of Experimental Psychology: General, 133, 189-217.

MORAY, N. (1959). Attention in dichotic listening: Affective cues and the influence of instructions. Quarterly Journal of Experimental Psychology, 11, 56-60.

Unsworth, N., Schrock, J. C., \& Engle, R. W. (2004). Working memory capacity and the antisaccade task: Individual differences in voluntary saccade control. Journal of Experimental Psychology: Learning, Memory, \& Cognition, 30, 1302-1321.

WoOD, N., \& CoWAN, N. (1995). The cocktail party phenomenon revisited: How frequent are attention shifts to one's name in an irrelevant auditory channel? Journal of Experimental Psychology: Learning, Memory, \& Cognition, 21, 255-260.

\section{NOTES}

1. The method of scoring WMC measures used by Conway et al. (2001) was different from that in the present experiment. Rescoring the WMC measures from the current experiment using the scoring procedure of Conway et al. yielded similar means and ranges for both the high $(M=21.93$; range of 16-30) and low spans $(M=8.05$; range of $1-13)$.

2 . The dichotic listening procedure of the present experiment was based on that used by Conway et al. (2001). In both experiments, the gender, the nature and the rate of presentation of the messages were the same. However, there were differences between the two studies, but the authors feel that they do not detract from interpretation of the results. There were three differences between Conway et al. and the present study: (1) the length of the messages (5.5 vs. 3 min, respectively); (2) the time of the presentation of the name (at 4 or 5 min vs. 1.5 or $2.5 \mathrm{~min}$, respectively); and (3) SNR (0 SNR vs. $-8,0$, and +8 SNR, respectively).

(Manuscript received April 27, 2006; revision accepted for publication November 7, 2006.) 